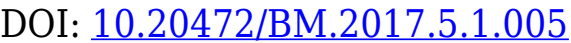

\title{
CONTROL STRATEGY TO TRADE CRYPTOCURRENCIES
}

\section{JOSEF KOKEŠ}

\section{Abstract:}

The paper deals with cryptocurrencies and trading. Main goal of this article is to introduce strategy for automated trading on cryptocurrency exchange market. For this purpose we will use algorithm based of Floyd-Warshall algorithm. Article is introductory and can this method can be developed in the future. First, a general introduction to cryptocurrencies is given from the programmer's point of view, some statistics data and figure representing volatility of exchange. Then the article describes some basic strategies for automated trading. Also explained is the algorithm Floyd-Warshall and its modifications for automation arbitrage. An illustrative example is given and a trading algorithm is listed.

\section{Keywords:}

cryptocurrency; arbitrage strategies; algorithm Floyd-Warshall

JEL Classification: C60, C80, G10

\section{Authors:}

JOSEF KOKEŠ, ČVUT, Czech Republic, Email: josef.kokes@gmail.com

\section{Citation:}

JOSEF KOKEŠ (2017). Control strategy to trade cryptocurrencies. International Journal of Business and Management, Vol. V(1), pp. 62-69., 10.20472/BM.2017.5.1.005 


\section{Introduction}

The paper deals with cryptocurrencies. Main goal of this article is to introduce strategy for automated trading. For this purpose we will use algorithm based of Floyd-Warshall algorithm. Article is introductory and can this method can be developed in the future.

\section{Introduction to cryptocurrencies}

Cryptocurrency is a type of digital currency that uses cryptography for security and anticounterfeiting measures. Cryptocurrency transactions are anonymous, and untraceable. For cryptocurrency enthusiasts, this anonymity is a preferred technology, as it empowers individuals rather than institutions. Cryptocurrencies typically feature decentralized control and a public ledger which records transactions. The first decentralized cryptocurrency became Bitcoin in 2009.

Bitcoin is a best known, distributed peer-to-peer digital currency that can be transferred instantly and securely between any two people in the world. It's like electronic cash that you can use to pay friends or merchants. Since its introduction in 2009, over 700 other artificial cryptocurrencies were invented, see (Antonopoulos, 2015). There were more than 669 cryptocurrencies available for trade in online markets as of 24 August 2015 (Farghaly, 2014) and more than 740 in total, but only 8 of them had market capitalizations over $\$ 10$ million (Farghaly, 2014).

Brief overview of top 15 cryptocurrencies with highest market capitalizations as of October 10, 2015 follows in Figure 2.

Typical properties of cryptocurrency include:

- decentralization - there is no central authority that could manage, control or influence cryptocurrencies. Processing of digital currency is based on the P2P networks, which are completely independent of any central server as well as on some specific hardware infrastructure. The con-sequence is that cryptocurrency cannot be controlled by the government or other institutions.

- transparency - the currency is fully transparent through public database called Blockchain that shows all transactions that were carried out on the network. Practically cannot be faked.

- anonymity - money transactions are carried out as a chain of digital signatures of individual trans-fers. This means that you can re-verify that means if a digital currency is right, but you cannot identify the sender. (Murphy, 2015)

- resistance against inflation - the number of units is determined by mathematical cryptocurrency addictions and cannot be changed. Some cryptocurrency (Bitcoin) therefore tend to have a deflationary character.

- $\quad$ non-refusing - one cannot refuse payment - sent the currency (e.g. Bitcoin) cannot cancel after you send or withdraw. Therefore there can be neither a chargeback by the bank or other institution.

For each of these characteristics, of course, one can lead a discussion, whether the property is convenient or inconvenient or even dangerous. 
It is worth saying that, compared with fiat currencies, all cryptocurrencies have much higher volatility and short-time volatility. On Figure 1 is a 15-minutes snapshot of trade (Bitcoin, Exchange OKCoin, November 1, 2015 at 14 o'clock). We can see that price varies from USD 332.3 to USD 307.48 and back, i. e. $+3.9 \%$ around its mean value.

It is therefore not uncommon (i.e. it is more the rule), that at the same time on different exchanges trades the same currency pair (for example, BCT / USD) at very different prices. From there it is very close to the idea, realized with cryptocurrency arbitrage. Taking into account the specific characteristics cryptocurrency however need to respect that arbitrage strategies will differ significantly from what we know of the standard exchanges, such as Ameritrade or Forex.

\section{Figure 1: Volatility of bitcoin exchange ${ }^{1}$}

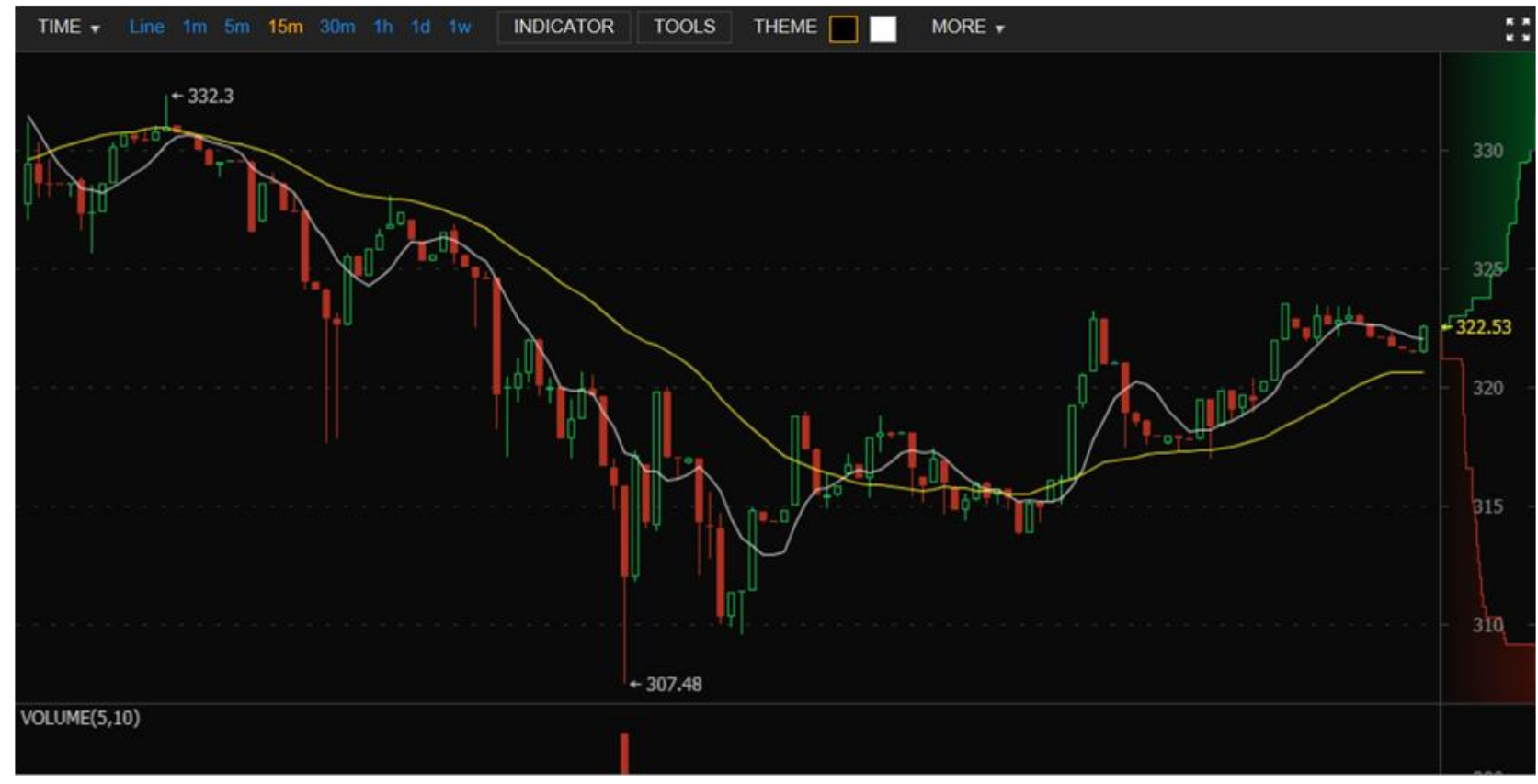

Basic arbitrage strategies, as we know from traditional exchanges, are risk-free. It constantly monitors the price range on the same stock, and whenever detects the business opportunities to buy cheaper one exchange and at the same time to sell for a higher price on other exchange. The difference between the sale and re-purchase price is (gross) profit transaction.

Why cryptocurrency exchange may work well in arbitration scheme involves far greater market volatility. At any change in prices other exchanges will adapt, but it's a process that takes place relatively slowly. Therefore, it is possible to use local fluctuations during fast changes in prices. (Gandal, 2014)

In naive approach we first buy the currency at a lower price (stage B), then this currency move (stage $\mathrm{T}$ for Transfer) to another exchange, and there sell it back (stage S) at a higher price. The whole cycle includes all three stages BST.

While high volatility on the one hand allows the use of arbitration strategy, on the other hand simultaneously is the limiting factor. While fiat currencies are forwarded mostly by hand, which

$1 \quad$ Khaosan (2014) 
takes several days, forwarding crypto currency is significantly faster and in theory can be done during milliseconds. Unfortunately, the transfer process includes verifying the authenticity of money. This is done by a mechanism called Blockchain and typically takes from tens of minutes to several hours. It is obvious that, given the rate of price changes, BST strategy is unusable.

Figure 2: Market Capitalizations of 15 Cryptocurrencies $^{2}$

\begin{tabular}{|c|c|c|c|c|c|c|}
\hline \# & Name & Market Cap & Price & $\begin{array}{l}\text { Available } \\
\text { Supply }\end{array}$ & $\begin{array}{l}\text { Volume } \\
(24 h)\end{array}$ & $\begin{array}{l}\% \\
\text { Change } \\
(24 h)\end{array}$ \\
\hline 1 & (3) Bitcoin & $\$ 3,503,103,376$ & $\$ 238.53$ & $\frac{14,686,525}{\underline{\text { BTC }}}$ & $\begin{array}{l}\$ \\
\underline{12,725,200}\end{array}$ & $-0.09 \%$ \\
\hline 2 & - Ripple & $\$ 171,340,417$ & $\$ 0.005274$ & $\frac{32,488,247,3}{\underline{36 \times R P}}$ & $\$ 339,598$ & $-6.89 \%$ \\
\hline 3 & CLitecoin & $\$ 128,324,751$ & $\$ 3.01$ & $\frac{42,576,510}{\underline{\mathrm{LTC}}}$ & $\$ 983,026$ & $-0.42 \%$ \\
\hline 4 & Ethereum & $\$ 49,141,111$ & $\$ 0.666966$ & $\frac{73,678,585}{\underline{\text { ETH }}}$ & $\$ 107,763$ & $-5.04 \%$ \\
\hline 5 & BitShares & $\$ 13,140,479$ & $\$ 0.005231$ & $\frac{2,511,953,11}{\underline{7 \mathrm{BTS}}}$ & $\$ 60,360$ & $-3.27 \%$ \\
\hline 6 & BanxShares & $\$ 13,019,479$ & $\$ 1.81$ & $\frac{7,174,808}{\text { BANX }}$ & $\$ 9,194$ & $-0.00 \%$ \\
\hline 7 & $\Xi_{\text {Dash }}$ & $\$ 12,482,092$ & $\$ 2.13$ & $\frac{5,849,153}{\underline{\text { DASH }}}$ & $\$ 36,899$ & $-1.63 \%$ \\
\hline 8 & (1) Dogecoin & $\$ 12,119,935$ & $\$ 0.000120$ & $\frac{101,248,360,}{146 \text { DOGE }}$ & $\$ 31,608$ & $-1.70 \%$ \\
\hline 9 & Stellar & $\$ 9,862,306$ & $\$ 0.002039$ & $\frac{4,837,356,60}{\underline{6 \mathrm{STR}}}$ & $\$ 1,062$ & $-2.62 \%$ \\
\hline 10 & MaidSafeCoin & $\$ 8,530,341$ & $\$ 0.018849$ & $\frac{452,552,412}{\text { MAID }}$ & $\$ 7,062$ & $0.49 \%$ \\
\hline 11 & Peercoin & $\$ 8,496,109$ & $\$ 0.374765$ & $\frac{22,670,499}{\underline{P P C}}$ & $\$ 6,945$ & $-0.01 \%$ \\
\hline 12 & S $\mathrm{Nxt}$ & $\$ 8,159,406$ & $\$ 0.008159$ & $\begin{array}{l}999,997,096 \\
\underline{N X T}\end{array}$ & $\$ 12,178$ & $-1.10 \%$ \\
\hline 13 & $\mathbf{B}_{\text {Bytecoin }}$ & $\$ 5,197,101$ & $\$ 0.000029$ & $\frac{176,581,137}{897 \mathrm{BCN}}$ & $\$ 9,191$ & $5.49 \%$ \\
\hline 14 & $\mathbb{N}_{\text {Namecoin }}$ & $\$ 4,637,428$ & $\$ 0.368272$ & $\frac{12,592,400}{\underline{N M C}}$ & $\$ 20,012$ & $-0.68 \%$ \\
\hline
\end{tabular}

2 Crypto-Currency Market Capitalizations (2015) 


\section{$3 \quad$ Naive strategy BST}

Uninformed investor may attempt to modify this basic strategy for cryptocurrency and the two exchanges. He reaches strategy, which we call naive, and that is totally bad. Numerical example of this strategy nicely describes article (Khaosan, 2014). Specifically, pure arbitrage in the Bitcoin exchange market is not viable because of the slow transaction mechanism imposed by the blockchain.

Pure arbitrage works well between mismatched futures and spot contracts because the same broker will accept immediate settlement of both sides of an arbitrage trade. Applying the principle of arbitrage to two currency accounts held at different exchanges is not only impractical - it is a catastrophic scenario.

Why cryptocurrency exchange may work well in arbitration scheme involves far greater market volatility. At any change in prices other exchanges will adapt, but it's a process that takes place relatively slowly. Therefore, it is possible to use local fluctuations during fast changes in prices. (Andrychowicz, 2015)

In naive approach we first buy the currency at a lower price (stage B), then this currency move (stage $\mathrm{T}$ for Transfer) to another exchange, and there sell it back (stage $\mathrm{S}$ ) at a higher price. The whole cycle includes all three stages BST. (Pieters, 2015)

While high volatility on the one hand allows the use of arbitration strategy, on the other hand simultaneously is the limiting factor. While fiat currencies are forwarded mostly by hand, which takes several days, forwarding crypto currency is significantly faster and in theory can be done during milliseconds. Unfortunately, the transfer process includes verifying the authenticity of money. This is done by a mechanism called Blockchain and typically takes from tens of minutes to several hours. It is obvious that, given the rate of price changes, BST strategy is unusable.

\section{$4 \quad$ Strategy BST}

Based on the analysis (Khaosan, 2014), I propose a new Buy-Sell-Transfer (BST) strategy. It is based on the fact that with simultaneous buying and selling currency. Under normal circumstances, this is not possible. Or, it would be only if we assume that on all accounts there is sufficient balance, respectively. But this is not useable in practice. (Kiran, 2014)

BST strategy uses so-called short sales. It is a feature available on many exchanges. Buying and selling short is, in fact, selling a commodity that we do not have yet. Does this mean that under certain circum-stances we may borrow from the Exchange for a short period of time. Later we must cover this borrow, including a small fee. Short time borrows ("selling short" in exchange terminology) is meant for different usage; however this can bring a substantial advantage.

The whole process begins by buying currency on one exchange for a small price. At the same time the same amount of currency is sold for higher price on other exchange. Later the currency, which we bought on the first exchange, is transferred onto second exchange and used to cover the loan. (Lema, 2015)

Because buying and selling currencies proceeds in exactly the same moment, prices may not change between selling and buying. BST strategy is inherently safe. Its weakness is, first, that the 
loan must be paid with a certain interest, but mainly the fact that many exchanges do not allow to simply cover the loan by the funds transferred.

As the purchase and sale occur simultaneously, and because it is a risk-free operation, comes into consideration even the situation that the purchase/sale proceeds via several exchanges. Thus, between the start and end Exchange is still one or several other middle exchanges.

This situation leads to the task of finding the optimal transition from the start, through one or more intermediate exchanges to the destination exchange. Tasks like this are dealt with in graph theory and can make a mathematically correct. There is a simple and effective algorithm that solves this task in $\mathrm{O}\left(\mathrm{N}^{3}\right)$. It's well known as Floyd-Warshall algorithm.

\section{$5 \quad$ Algorithm Floyd-Warshall}

Finding the optimal path can be solved using a modified algorithm Floyd-Warshall.

Original, unmodified algorithm is explained on Figure 2. It is closely related to Kleene's algorithm (published in 1956) for converting a deterministic finite automaton into a regular expression.

According to (Ciubatii, 2010), the algorithm can be divided into two steps:

1. Let $A$ be a NxN matrix ( $N$ is the number of vertices), $A(i, j)$ representing the length (or cost) of the shortest path from $i$ to $j$. For each element $A(i, j)$ assign a value equal to the cost of the edge going from $i$ to $j$, or an infinity value if this edge doesn't exist.

2. At each step, for each pair of vertices $i$ and $j$ see if there's an intermediate vertex $k$ so that the path from $i$ to $j$ through $k$ is shorter than the one already found for $i$ and $j$. If $i$, $j$ and $k$ are or-dered properly, only $\mathrm{O}\left(\mathrm{N}^{3}\right)$ operations are needed to find the values of all elements of $A$. Such an order is obtained when first $k$ is considered and then $i$ and $j$.

Figure 3: Example of graph and matrix for graph

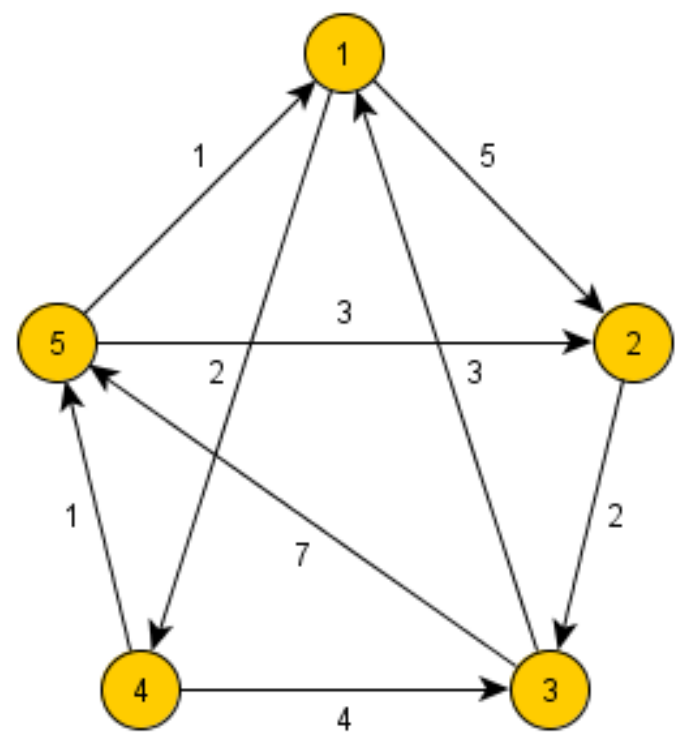

$$
\left(\begin{array}{ccccc}
0 & 5 & \infty & 2 & \infty \\
\infty & 0 & 2 & \infty & \infty \\
3 & \infty & 0 & \infty & 7 \\
\infty & \infty & 4 & 0 & 1 \\
1 & 3 & \infty & \infty & 0
\end{array}\right)
$$


Figure 4: Pseudocode for Floyd-Warshall Algorithm ${ }^{3}$

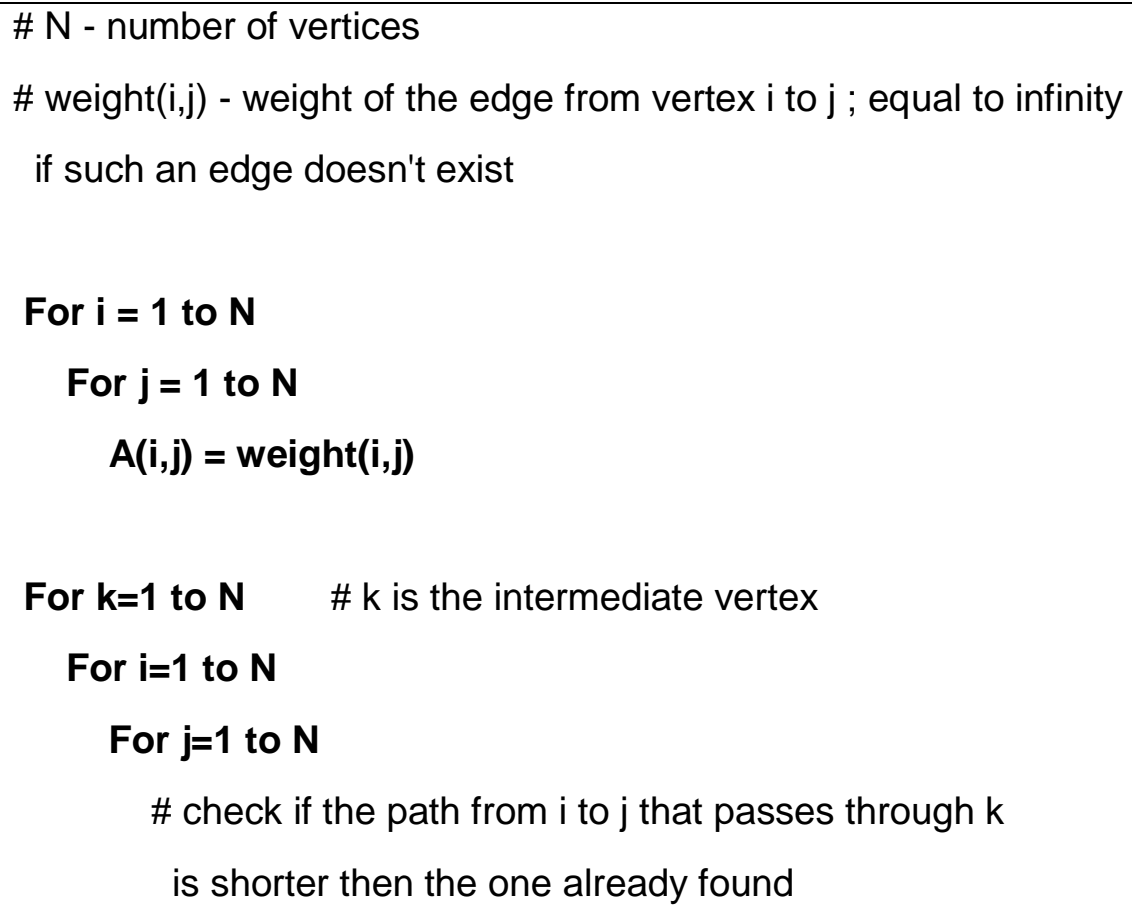

\section{Modification of Floyd-Warshall Algorithm}

Described algorithm cannot be used in its original form. It needs to be modified, at least for the following reasons:

1. The original algorithm assumes that the prices of the trip are additive, i.e. when passing through the multiple edges, the individual contributions are summed. In contrast, in the search for the optimum way through graph of exchanges are gains for the individual edges multiplicative, i.e. profits of edges are multiplied.

2. Unlike classical algorithm, we do not seek to minimize prices, but rather to maximize profits. This is why comparison of profits must be held in more sophisticated way.

3. Algorithm Floyd-Warshall assumes a uniform scale for prices. This assumption in practical use is not met, because there is no clearly defined reference point. Simply put, when you buy or sell you cannot simply decide whether the trade is profitable or not. It depends on against which the cur-rency and against which exchange the profitability is compared.

4. There is a whole range of other restrictions that are enforced both by internal regulations and the implementation of the API in exchanges. For example, you cannot make trades between two fiat currencies (legislative restrictions: because exchanges do not have a

Ciubatii (2010) 
banking license for currency exchange) and the like. More detailed analysis would, however, be beyond the contribution.

\section{Conclusion}

The article provides a brief insight into cryptocurrencies. It deals with one interesting aspect arbitrage and brings a new strategy BST for automated trading.

In the second part the article discusses the possible application of Floyd-Warshall algorithm for finding the optimum path through the graph so that profit is maximized. There are described some problems of implementation and outlined ways to solve them. But there is still a lot of work to do. More testing on real data should be done.

\section{References}

Algoritmy.net. (2015). Floyd-Warshallův algoritmus. Retrieved November 01, 2015, from https://www.algoritmy.net/article/5207/Floyd-Warshalluv-algoritmus

Andrychowicz M., Dziembowski S., Malinowski D., Mazurek Ł. (2015) On the Malleability of Bitcoin Transactions. In: Brenner M., Christin N., Johnson B., Rohloff K. (eds) Financial Cryptography and Data Security. FC 2015. Lecture Notes in Computer Science, vol 8976. Springer, Berlin, Heidelberg. https://doi.org/10.1007/978-3-662-48051-9_1

Antonopoulos, A. M. (2015). Mastering bitcoin: Unlocking digital cryptocurrencies. O'Reilly Media.

Ciubatii, D. (2010). Shortest Paths between every pair of vertices. Retrieved November 01, 2015, from http://www.graph-magics.com/articles/all_shortest_paths.php

Crypto-Currency Market Capitalizations. (2015). Retrieved September 24, 2015, from http://coinmarketcap.com/

Farghaly, M. (2014). Bitcoin Programming.

Gandal, V., Hałaburda, H. (2014) Competition in the Cryptocurrency Market, Bank of Canada Working Paper 2014-33, 2014

Kelly, B. (2014). Bitcoin big bang: How alternative currencies are about to change the world.

Khaosan, V. (2014). Bitcoin Price Arbitrage Explored - CCN: Financial Bitcoin \& Cryptocurrency News. Retrieved October 25, 2015, from https://www.cryptocoinsnews.com/bitcoin-price-arbitrage-explored

Kiran, M., Stannett, M. (2014) BitcoinRisk Analysis

Lema, P. (2015) Crypto Success: Investing in Cryptocurrency for the Long Term - Tips and Tricks

Map of coins: The history of cryptocurrencies from bitcoin to dogecoin and more. (2015). Retrieved November 03, 2015, from http://mapofcoins.com/

Murphy, V. et al. (2015) Bitcoin: Questions, Answers, and Analysis of Legal Issues, Congressional Research Service

Pieters, G., Vivanco, S. (2015) Bitcoin arbitrage and unofficial exchange rates

Vigna, P., \& Casey, M. (2015). The age of cryptocurrency:how bitcoin and digital money are challenging the global economic order. New York: St. Martin's Press. 